\title{
O trabalho pedagógico e a inclusão escolar para crianças com transtorno do espectro do autismo (TEA)
}

\author{
Pedagogical work and school inclusion for children with autism spectrum disorder (ASD) \\ Trabajo pedagógico e inclusión escolar para niños con trastorno del espectro autista (TEA)
}

Recebido: 06/10/2021 | Revisado: 11/10/2021 | Aceito: 16/10/2021 | Publicado: 18/10/2021

Laila Francielly Rezende

ORCID: https://orcid.org/0000-0001-6277-6478 Instituto Federal de Educação, Ciência e Tecnologia Goiano, Brasil

E-mail: lailafran1979@gmail.com

Calixto Júnior de Souza

ORCID: https://orcid.org/0000-0003-3591-1173 Instituto Federal de Educação, Ciência e Tecnologia Goiano, Brasil E-mail: calixto.souza@ifgoiano.edu.br

\begin{abstract}
Resumo
O objetivo deste trabalho é mostrar uma visão geral do autismo, bem como sinalizar para a inclusão do aluno autista em escolas regulares, discutindo concepções equivocadas, tais como a de que alunos com autismo são pessoas que não conseguem de forma alguma se comunicar ou alcançar progressos intelectuais; buscando explicar as causas do comportamento singular dessas pessoas, e que a inclusão é possível e deve ser realizada de forma especializada. O autismo é caracterizado por dificuldades na comunicação, socialização e por comportamentos repetitivos ou restritos. A inclusão de crianças diagnosticadas com Espectro do Autismo (TEA) no ambiente escolar se faz necessária e deve partir de uma concepção de formação de professores especializados no atendimento desse público, bem como por meio do estabelecimento das condições necessárias ao desenvolvimento do sujeito autista, que vão desde aspectos estruturais das instituições de ensino até a elaboração de políticas públicas específicas. O TEA é um transtorno que afeta as áreas de interação social, comunicação/linguagem e comportamento, podendo acometer a criança de forma leve, moderada ou severa. Atualmente as intervenções utilizadas para promover o desenvolvimento e a aprendizagem desse público são: TEACCH, ABA e PECS. É válido destacar que o autismo é um transtorno complexo, portanto não existe uma terapia ou método isolado que atenderá as demandas da pessoa com TEA. É preciso fortalecer os vínculos entre família, escola, cuidadores e terapeutas para que deste modo, juntos possam criar uma melhor estratégia de intervenção para o ensino de habilidades que vise melhorar o desenvolvimento da criança.
\end{abstract}

Palavras-chave: Docente; Educando; Inclusão; Transtorno do Espectro Autista.

\begin{abstract}
The aim of this paper is to show an overview of autism, as well as to signal for the inclusion of the autistic student in regular schools, discussing misconceptions, such as that students with autism are in no way able to communicate or achieve progress intellectuals; seeking to explain the causes of the singular behavior of these people, and that inclusion is possible and should be carried out in a specialized way. Autism is characterized by difficulties in communication, socialization and repetitive or restricted behaviors. The inclusion of children diagnosed with ASD in the school environment is necessary and must start from a conception of training teachers specialized in serving this public, as well as by establishing the necessary conditions for the development of the autistic subject, which range from structural aspects of children with autism. educational institutions until the elaboration of specific public policies. ASD is a disorder that affects the areas of social interaction, communication / language and behavior, and may affect the child in a mild, moderate or severe manner. Currently the interventions used to promote the development and learning of this audience are: TEACCH, ABA and PECS. It is worth noting that autism is a complex disorder, so there is no isolated therapy or method that will meet the demands of the person with ASD. It is necessary to strengthen the bonds between family, school, caregivers and therapists so that, together, they can create a better intervention strategy for teaching skills aimed at improving the child's development.
\end{abstract}

Keywords: Teacher; Teaching; Inclusion; Autistic Spectrum Disorder.

\section{Resumen}

El objetivo de este trabajo es mostrar una visión general del autismo, así como señalar la inclusión del estudiante autista en las escuelas regulares, discutiendo conceptos erróneos, como que los estudiantes con autismo son personas que no pueden comunicarse o progresar en absoluto. intelectuales; buscando explicar las causas del comportamiento singular de estas personas, y que la inclusión es posible y debe realizarse de forma especializada. El autismo se caracteriza por dificultades en la comunicación, socialización y conductas repetitivas o restringidas. La inclusión de 
los niños diagnosticados con TEA en el ámbito escolar es necesaria y debe partir de un concepto de formación de docentes especializados en la atención a este público, así como mediante el establecimiento de las condiciones necesarias para el desarrollo del sujeto autista, que van desde aspectos estructurales. de las instituciones educativas a la elaboración de políticas públicas específicas. El TEA es un trastorno que afecta las áreas de interacción social, comunicación / lenguaje y comportamiento, y puede afectar al niño de manera leve, moderada o severa. Actualmente, las intervenciones utilizadas para promover el desarrollo y aprendizaje de esta audiencia son: TEACCH, ABA y PECS. Vale la pena señalar que el autismo es un trastorno complejo, por lo que no existe una terapia o método aislado que satisfaga las demandas de la persona con TEA. Es necesario fortalecer los lazos entre familia, escuela, cuidadores y terapeutas para que, juntos, puedan crear una mejor estrategia de intervención para la enseñanza de habilidades orientadas a mejorar el desarrollo del niño.

Palabras clave: Inclusión; Trastorno del Espectro Autista; Maestro; Enseñando.

\section{Introdução}

Os estudos científicos acerca do autismo tiveram início na década de 1940, mais precisamente no ano de 1942 com os trabalhos de Leo Kanner (1894-1981), que descreveu o autismo sob o nome de "distúrbios autísticos do contato afetivo", caracterizado por um quadro de autismo extremo, ecolalia, estereotipias e obsessividade, sintomas relacionados à linha da esquizofrenia. Mais tarde foi descrito pelo mesmo autor como "um quadro de psicose", devido à inconsistência dos dados laboratoriais e clínicos que fornecessem uma resposta a que se relacionava sua etiologia. A partir de estudos e pesquisas (Kupfer, 2000; Santos, Lemes, 2020), o autismo deixou de ser classificado como psicose, e passou a ser classificado como um distúrbio do desenvolvimento.

O autismo representa um grande desafio para áreas como a psiquiatria, psicologia e psicopedagogia, além de outros campos profissionais da saúde. O diagnóstico é complexo, e, ao contrário do que se percebe empiricamente no imaginário social, não são todos os autistas que possuem deficiência mental. Estudos apontam que a pessoa com TEA apresenta grandes avanços no seu desenvolvimento quando são realizadas intervenções precoces, sistematizadas e individuais, minimizando os comportamentos exacerbados e estereotipias, aumentando assim sua independência e autonomia.

Uma revisão de oito programas de intervenção precoce para crianças autistas entre três e meio e quatro anos de idade, realizada por Dawson e Osterling (1997), conclui que todos foram eficazes em proporcionar a colocação de 50\% das crianças em uma escola regular. Segundo os autores, para assegurar um resultado positivo, certos aspectos fundamentais devem estar presentes. Os elementos comuns desses programas foram: currículo abrangendo cinco habilidades (prestar atenção a elementos do ambiente, imitação, compreensão e uso da linguagem, jogo apropriado com brinquedos e interação social), ambiente de ensino altamente favorecedor e estratégias para a generalização para ambientes naturais, programas estruturados e rotina, abordagem funcional para comportamentos considerados problemáticos, transição assistida para a pré-escola (Lampreia, 2007, p. 105).

O autista apresenta alterações nas interações sociais recíprocas e seus interesses na maioria das vezes são restritos, estereotipados e repetitivos, apresentando dificuldades de aprendizagem, podendo estar ou não associadas a limitações biopsicossociais. O apoio familiar conjugado com o apoio da escola apresenta incontáveis benefícios para o autista, tanto social, como emocional e cognitivo (Butet; Godinho, 2017).

É importante pontuar que o atendimento à pessoa com TEA deve ser interdisciplinar, conforme descreve Facion (2007), pois o trabalho interdisciplinar, realizado de forma integrada pelos profissionais das áreas envolvidas, deve priorizar ações e técnicas comuns, que precisam ser dialogadas com pais, destacando as necessidades das crianças quanto ao que conseguem entender e executar. O paradigma da incapacidade da pessoa com TEA é fundamental para o processo da inclusão. Sabe-se que há grande resistência por parte de alguns educadores, devido aos padrões impostos por uma visão equivocada e ultrapassada, que exclui qualquer um que não se enquadre nos padrões de normalidade impostos.

Os professores, com a prática, aprendem a lidar com o aluno com deficiência, porém, não contam com o conhecimento teórico que apoie essa prática. Professores de educação infantil que têm contato direto com o processo 
de inclusão, ressaltam que as principais dificuldades são com o espaço físico, recursos materiais e humanos e relativos à sua própria formação (Pimentel; Fernandes, 2014, p. 172).

O autista não é um deficiente, mas sim um indivíduo com Transtorno Global do Desenvolvimento, e pode ser beneficiado com a inclusão escolar, pois esta requer um mínimo de capacidades intelectuais da criança, bem como capacidades sensoriais e motoras (Marinho, 2009).

É fundamental conhecer o autismo, suas características e especificidades, e as possíveis intervenções pedagógicas para que se possa realizar um trabalho de inclusão verdadeira, duradouro e que traga respostas significativas no aprendizado do autista. Apesar do grande espaço que há na literatura para discussões acerca da inclusão, percebe-se na prática docente muitas barreiras que ainda precisam ser derrubadas, como o preconceito, medos, dúvidas e a ignorância. Dessa forma, este trabalho almeja responder o seguinte questionamento: Como o trabalho pedagógico pode ajudar no processo de ensino e de aprendizagem das crianças com TEA (Transtorno do Espectro do Autismo)? Além disso, pretende-se discutir uma visão geral sobre o TEA, suas características e intervenções pedagógicas que promovem o desenvolvimento da criança, bem como sua inclusão, desmistificando as crenças e limitações equivocadas que justificam o comportamento peculiar e ímpar das pessoas com o transtorno do espectro autista.

\section{Metodologia}

Utilizou-se o estudo de caso na pesquisa descritivo e como procedimento metodológicos para execução do estudo adotou-se a pesquisa bibliográfica (Pereira et al. 2018) com enfoque nos estudiosos Lampreia (2007); Matsumoto; Campos (2008); Pletsch (2010); Mello (2011); Cunha (2012); Balestro et al. (2012); Baptista; Bosa (2012); Bosa; Callias (2012); Papim; Sanches (2013); Ziraldo (2013); Fernandes (2014); Aires (2014); Almeida; Felizardo (2015); Caneda; Chaves (2015); Kanner (2015); Leboyer; Marion (2015); Uchôa (2015); Leboyer; Marion (2015); Glat; Nogueira (2016); Leon (2016); Assumpção; Pimentel (2017); Benitez et al. (2017); Burtet; Godinho (2017); Pimentel; Santos; Lemes (2020) visando trabalhar o autismo bem como a sinalização para inclusão do aluno autista nas escolas. A averiguação e a busca de artigos em periódicos, trabalhos de conclusão de curso, dissertações e teses foram realizados nas bases de dados SciELO, Scopus, Web of Science e Google acadêmico com abordagens sobre o assunto em questão. Como palavras-chave foi utilizada "inclusão", "Transtorno do Espectro Autista" e "trabalho pedagógico para autistas" em português e "inclusion", "Autistic Spectrum Disorder" and "pedagogical work for autistics" em inglês

\section{Autismo: Definição e Conceito}

O autismo é um distúrbio do desenvolvimento, sendo caracterizado por alterações que surgem antes dos três anos de idade, cujo impacto na vida da criança é múltiplo e muito variável em áreas do desenvolvimento humano, tais como a capacidade de adaptação, comunicação, interação social e aprendizado. O distúrbio foi descrito pela primeira vez por um médico austríaco, residente em Baltimore, nos EUA em 1943, chamado Leo Kanner, em um artigo intitulado: Distúrbios Autísticos do Contato Afetivo, publicado na revista Nervous Children (1943, p. 217-250). Na publicação, Kanner (1943) descreveu casos de onze crianças que apresentavam alto grau de isolamento social desde o início da vida, além de um anseio obsessivo pela preservação da rotina, denominando-as de "autistas". A obsessão pelo isolamento, presente desde o início da vida da criança, apontava, até então, para um distúrbio inato (Leboyer, 2015).

Em 1944, outro médico, Hans Asperger, também austríaco, descreveu casos de crianças muito semelhantes às mencionadas por Kanner. Atualmente, a definição do autismo, é atribuída tanto a Kanner como a Asperger (MELLO, 2011). O autismo foi identificado na década de 1940. Sendo o seu diagnóstico complexo, e devido o assunto ser inesgotável, novas 
descobertas surgiram, permanecendo em constante avaliação.

Segundo Ziraldo (2013), o autismo é uma característica que já nasce com o sujeito, caracterizado como um transtorno neurobiológico, o que afeta o desenvolvimento cognitivo e faz com que tenha dificuldades no relacionamento social e na interação com o ambiente. Necessitando, assim, de ajuda para se desenvolver e superar suas limitações. De acordo com Bosa (2002), as crianças autistas apresentam inadaptações para manter relações sociais com outros sujeitos, atraso na aquisição da linguagem e, quando ela se desenvolve, dificuldade em lhe atribuir valor de comunicação. Estudos recentes têm mostrado que o autismo é quatro vezes mais frequente no gênero masculino, e incide de forma equivalente em indivíduos de diferentes etnias ou classes sociais. Diversas metodologias vêm sendo usadas por vários países para avaliar a incidência do autismo (Mello, 2011). A gravidade do autismo é fundamentada em alguns princípios que demandam ações que guardem as devidas orientações para que as interações sociais sejam estabelecidas.

Segundo destaca APA - American Psychiatric Association (2014), a gravidade do autismo acontece em três níveis, conforme seu comprometimento, podendo ser considerado: leve, moderado ou severo. O nível 1 (leve), necessita de pouco suporte, pode ter déficit para se comunicar, o que não implica nas interações sociais, apesar de demonstrar dificuldade em iniciá-las. Apresenta, também, dificuldade de organização e planejamento. O nível 2 (moderado), apresenta acentuado transtornos de comunicação social, verbal e não verbal, deficiência de linguagem, além de restrições na interação, comportamento, organização e planejamento. No nível 3 (severo), é verificado déficit grave nas habilidades de comunicações verbais e não verbais, além de comprometimento severo nas interações sociais e cognitivas com tendências ao isolamento, apresentando também comportamento inflexível, restrito/repetitivo e dificuldades em lidar com mudanças da rotina. Diante disso, percebe-se que os Transtornos do Espectro do Autismo estão fundamentados essencialmente em uma condição presente desde o nascimento, que afeta as habilidades de socialização, comunicação e comportamento, podendo acometer a criança de forma leve, moderada ou grave.

\subsection{A inclusão do aluno autista}

Ao tratar da inclusão do aluno autista, é comum pensar, simplesmente, em inserir a criança autista em uma escola regular, acreditando que ela imitará as demais, adequando seu comportamento aos modos coletivos esperados. As crianças com autismo, quando pequenas, dificilmente imitam outras crianças. A imitação ocorre quando o autista começa a ter consciência de si mesmo, percebendo relações de causa e efeito do ambiente em relação a suas próprias ações. A inclusão do autista é controversa, pois muitos acreditam que essas crianças são completamente desprovidas de inteligência e capacidades diversas, não sendo essa a realidade. Um programa de intervenção e uma adequada estratégia de ensino pedagógico podem contribuir positivamente para minimizar os impactos dos TEA, favorecendo o desenvolvimento do sujeito. Segundo Mazzota (2009) a prática da inclusão passou a ser discutida devido a necessidade de inserir e garantir às pessoas com deficiência, o direto a educação, o que demandou a criação de políticas educacionais inclusivas.

A atividade de inclusão para o TEA exige que se tenha o compromisso e a determinação, bem como vigência de novos olhares quanto ao que atende e orienta na formação educacional e social. A educação ainda representa a possibilidade de transformação da sociedade. $\mathrm{O}$ alicerce para essa integração é a escola, as políticas públicas que garantam tal implantação baseada em Leis que lutam pela igualdade e pelo direito à educação para todos, como a LDB/96 que assegura o direito às pessoas com necessidades educacionais especiais em seu capítulo $\mathrm{V}$, artigo 58 no inciso $1^{\circ}$, também no artigo 59.

Alguns autistas levam tempo para adquirir consciência sobre si mesmos, outros podem nunca vir a desenvolvê-la. Antes da inclusão numa escola regular, o ideal é que a criança seja preparada por meio de um atendimento especializado, para que esta adquira uma consciência em relação às suas potencialidades, antes de tentar a inclusão (Mello, 2011). A presença da criança com autismo na escola regular é um grande avanço. Contudo, a ação por si só não é garantia de aprendizado, sendo 
necessário o acompanhamento de um profissional especializado com formação adequada, com estratégias de ensino adequadas, para que haja êxito no processo de inclusão. É fundamental distinguir os profissionais que atuam no ensino regular daqueles que trabalham com o ensino especializado, voltado para a educação inclusiva. A formação de professores deve ser pensada com o professor e não para o professor, com a intenção de trazer ganhos para o aluno por meio da educação (Glat; Nogueira, 2016).

Projetos pedagógicos definidos e estruturados requerem planejamento, estudo de características comuns aos alunos autistas, e das particularidades de cada indivíduo atendido pelo professor de ensino especial, para que a inclusão se proceda de maneira satisfatória e adequada. O professor deve dedicar total atenção ao aluno a fim de interagir e se comunicar com ele, atender suas necessidades educacionais para que uma evolução, tanto do aluno quanto do professor, aconteça de forma mútua ao longo do processo de ensino e aprendizagem. Cada autista é único, e responde de forma diferente às intervenções, além de ter seu próprio tempo, diferente das demais crianças. À medida que a inclusão é realizada, é notável o desenvolvimento do autista em seu processo de aprendizagem e desenvolvimento, principalmente no que diz respeito à socialização e linguagem (Pletsch, 2010).

O Decreto n. 7.611/2011 que dispõe sobre a educação especial e inclusiva destaca que, o atendimento educacional especializado, mostra orientações para a educação especial, uma vez que estabelece como responsabilidade do Estado a:

I - garantia de um sistema educacional inclusivo em todos os níveis, sem discriminação e com base na igualdade de oportunidades;

II - aprendizado ao longo de toda a vida;

III - não exclusão do sistema educacional geral sob alegação de deficiência;

IV - garantia de ensino fundamental gratuito e compulsório, asseguradas adaptações razoáveis de acordo com as necessidades individuais;

V - oferta de apoio necessário, no âmbito do sistema educacional geral, com vistas a facilitar sua efetiva educação;

VI - adoção de medidas de apoio individualizadas e efetivas, em ambientes que maximizem o desenvolvimento acadêmico e social, de acordo com a meta de inclusão plena;

VII - oferta de educação especial preferencialmente na rede regular de ensino;

VIII - apoio técnico e financeiro pelo Poder Público às instituições privadas sem fins.

Segundo Gauderer (1987, apud Aires et al., 2014, p. 3), as crianças com autismo, em geral, apresentam dificuldades em aprender a utilizar corretamente as palavras, mas quando participam de um programa intenso de aulas parecem ocorrer mudanças positivas nas habilidades de linguagem, motoras, interação social e a aprendizagem. Neste contexto, fica evidente a importância desta inclusão ser bem estruturada, para garantir o sucesso no processo de desenvolvimento dessas crianças/educandos.

\subsection{O trabalho pedagógico para autistas}

Atualmente existem métodos de intervenção educacional para atender pessoas com TEA, que atuam visando desenvolver habilidades de interação social, comunicação e comportamento. O ensino escolar para os alunos com TEA representa um desafio contemporâneo para os educadores e familiares, uma vez que as alterações neuropsiquiátricas afetam as áreas de comunicação e comportamental (Almeida; Felizardo, 2015). Uma vez diagnosticado o autismo em uma criança, esta deve ser imediatamente submetida a uma intervenção educacional.

Ao estabelecer meios de comunicação convencionais, as formas como os autistas expressam suas necessidades não são compreendidas de imediato, e os métodos educacionais empenhados em desenvolver a socialização, tornam menos acentuadas as dificuldades que o autista tem em se comunicar, desenvolvendo uma linguagem melhor, facilitando a comunicação social (Babtista; Bosa, 2012). Os métodos educacionais são empenhados em desenvolver a socialização, comunicação e comportamento das pessoas com TEA: TEACCH, ABA e PECS. 


\section{Tratamento em Educação para Autista e Crianças com Deficiências Relacionadas à Comunicação}

TEACCH, em português significa Tratamento e Educação para Crianças com Autismo ou Desordem Relacionadas à Comunicação. Trata-se de um método que busca observar os comportamentos das crianças com TEA em situações distintas e ajustar as estratégias de intervenção. O TEACCH foi desenvolvido pelo Departamento de Psiquiatria da Faculdade de Medicina da Universidade da Carolina do Norte, EUA, nos anos 1960, e é muito usado em diversas partes do mundo. Sua idealização e desenvolvimento foram realizadas pelo Dr. Eric Schoppler. Esse método utiliza uma avaliação chamada PEP-R (Perfil Psicoeducacional Revisado), que serve para avaliar o autista considerando seus pontos fortes e suas principais dificuldades, tornando possível uma intervenção individualizada. (Gomes; Silva, 2007, p.89).

O método se baseia na organização do ambiente físico por meio de rotinas organizadas em quadros, painéis ou agendas e sistemas de trabalho, de forma a adaptar o ambiente para tornar mais fácil para a criança compreendê-lo, assim como compreender o que se espera dela. Através da organização do ambiente e das tarefas da criança, o TEACCH visa desenvolver a independência da criança de modo que ela necessite do professor para o aprendizado, mas que possa também passar grande parte de seu tempo ocupando-se de forma independente (Mello, 2005, p.28).

Este método se baseia, na programação individual de cada aluno, dando previsibilidade, confiança e segurança ao autista em realizar as atividades do cotidiano escolar, bem como da sua rotina pessoal, indicando visualmente as tarefas a serem realizadas, procurando melhorar a qualidade de vida do indivíduo, respeitando suas limitações e individualidade. Com base em Leon (2016), o TEACCH tem nos mostrado, nesses 40 anos, que toda criança com TEA, se atendida precoce e adequadamente, tem muitas chances de se desenvolver e de poder contribuir com a nossa sociedade e comunidade, tornando-se um adulto produtivo e independente. É essa a grande mensagem que o TEACCH nos deixa: "Intervenção adequada, intervenção precoce, promoção da independência, produção da produtividade, promoção à saúde”.

\section{Análise do Comportamento Aplicada}

ABA é uma sigla da língua inglesa que significa Applied Behavior Analysis, ou, em português, Análise do Comportamento Aplicada. É uma ciência que atua na intervenção das variáveis que afetam os comportamentos. É uma abordagem utilizada desde a década de 1960 para o tratamento de pessoas com TEA. Mostra que o planejamento fundamentado em intervenções variadas e individuais, pode contribuir para o desenvolvimento das habilidades da pessoa com TEA.

A terapia ABA envolve o ensino intensivo e individualizado das habilidades necessárias para que o indivíduo possa adquirir independência e melhor qualidade de vida. Dentre as habilidades ensinadas incluem-se comportamentos sociais, tais como contato visual, comunicação funcional e comportamentos acadêmicos, tais como pré-requisitos para leitura, escrita e matemática; além de atividades da vida diária como higiene pessoal. O reforço faz parte de seu processo, assim, a recompensa torna viável para alcançar os objetivos propostos.

ABA: Análise aplicada ao comportamento. O objetivo do tratamento comportamental analítico do autismo é ensinar a criança habilidades que ela não tem, através de etapas, individualmente, e a princípio, apresentando essas habilidades associadas a uma instrução, oferecendo algum apoio se necessário, porém retirado assim que possível para não deixar que a criança se torne dependente dele. O ponto mais importante e que a aprendizagem seja agradável, o segundo ponto é ensinar a criança a identificar os diferentes estímulos (Gomes; Silva, 2007, p.89).

Desta forma, pode-se constatar que o ensino da linguagem aos autistas, deve ser realizado em ambientes naturais da criança, facilitando a rotina e permitindo que eles possam responder melhor aos estímulos (Assumpção, 2017). 


\section{Sistema de Comunicação por Troca de Figuras}

A troca de figuras para que o aluno identifique tais alterações constitui uma soma de ações para entender o autismo. Assim, é fundamental e eloquente cada momento da prática e a efetividade da aprendizagem em todo processo de desenvolvimento e aprimoramento do aluno. O intercâmbio das figuras é uma forma alternativa de comunicação.

PECS: Sistema de comunicação através da troca de figuras. O PECS foi desenvolvido para auxiliar tento crianças como adultos autistas, e pessoas com outros distúrbios de desenvolvimento a adquirem habilidades de comunicação. Inicialmente o sistema é usado com pessoas que conseguem se comunicar ou que possuem capacidade de se comunicarem, porém com baixa eficiência. O objetivo do PECS é ajudar a criança a se comunicar mostrando que dessa forma pode conseguir com mais rapidez aquilo que deseja, estimulando-a a comunicar-se. Existem diversas outras formas de tratamento, como os psicoterapêuticos, fonoaudiólogos, e outros como equoterapia e musicoterapia, que não possuem uma linha formal para que sejam caracterizados como tratamento do autismo, porém dependem diretamente da visão, dos objetivos e do bom senso de cada profissional que os aplica (Gomes; Silva, 2007, p. 89).

A utilização do PECS foi adaptada por meio de desenhos, clip-arts, encartes ou figuras de softwares específicas ou sites que contenham pictogramas de comunicação alternativa. Os cartões de comunicação contendo o sistema gráfico determinado devem sempre vir acompanhados pela escrita (legenda) na parte superior. Os cartões com as imagens podem ser impressos em preto e branco ou em cores, plastificados, e devem conter no verso um pequeno pedaço de velcro para permitir que sejam fixados nos painéis ou no álbum de comunicação. A troca do cartão representa a comunicação.

As metodologias de intervenção educacional: TEACCH, ABA e PECS, são respectivamente recursos de cunho visual, lúdico e interativo, proporcionando ao aluno com TEA possibilidades de aprendizagem, interação, comunicação e regulação de comportamentos. Nesse sentido é importante considerar a vivência dos alunos, suas particularidades, para a partir daí traçar um plano de intervenção pedagógica individualizado.

[...] "ao usar a ideia de um programa diário visual individual, e fazê-la conter somente atividades enfadonhas que os alunos já conhecem, sempre apresentadas na mesma ordem. Assim a ideia perde sua função para a pessoa envolvida. Temos de pensar no que poderia interessante para ele, de forma que os conteúdos do dia sejam um acordo entre as coisas que julgamos que ele precisa fazer e coisas que ele prefere fazer" (Nilsson, 2014, p. 57).

De acordo com a APA (2014), o indivíduo com TEA apresenta desenvolvimento, comprometido anormal da inserção social e da comunicação e um repertório muito restrito de atividades e interesses com atrasos no comportamento social e linguagem, o que dificulta o aprendizado e interação, sendo um trabalho pedagógico de grande representatividade e mudanças quanto ao seu comportamento e aprimoramento das atividades propostas. Assim, as atividades vão ao encontro das necessidades do educando.

Papim e Sanches (2013) relatam que o método mais utilizado para trabalhar com alunos com autismo é a Análise Aplicada do Comportamento (ABA), a qual se utiliza da avaliação comportamental para ajudar, não só no comportamento, mas, para estabelecer a base para que a aprendizagem aconteça, sendo que cada etapa é contextualizada para o desempenho de uma aprendizagem voltada para a prática da aprendizagem do educando.

As diferentes formas de abordagem das atividades pedagógicas permitem o processo de inclusão e determinação do que de fato considera a participação do educando. A atividade individual é fundamental para o professor observar o desempenho do aluno em sala e o seu grau de aprendizagem, já as atividades em grupo favorecem a troca de informações entre os alunos, onde o educando aprende a ouvir, esperando a sua vez de falar, bem como construir o conhecimento coletivamente, conforme explanam Matsumoto e Campos (2008), enfatizando que o aprendizado em grupo é fundamental para o desenvolvimento cognitivo e social do aluno.

A contextualização do social é a principal fonte de determinação para com o educando e os resultados que emanam 
cada desenvolvimento e medidas para a inclusão dos trabalhos pedagógicos no ambiente escolar são os principais focos para tal exercício.

Uchôa (2015) assinala que apesar das dificuldades, os educadores devem proporcionar oportunidades iguais aos alunos para que as crianças com autismo sejam aceitas pela turma, valorizando-as e aceitando as suas limitações e respeitando as suas diferenças. $\mathrm{O}$ ambiente da sala de aula com equivalência para as atividades, auxiliam no trabalho do autista, pois é preciso garantir e propiciar o reconhecimento de cada um ao seu meio, criando estratégias e soluções que atendam e facilitem a aprendizagem de cada indivíduo mediante as suas perspectivas e potencialidades.

Ainda que o aluno não aprenda perfeitamente o que se busca ensinar, ele exercitará sempre a interação, a comunicação, a cognição e os movimentos. Haverá conquistas e erros, muitas vezes mais erros do que conquistas, mas o trabalho jamais será em vão (Cunha, 2012). É fundamental que o currículo aborde questões a respeito da prática pedagógica, nas quais o professor trabalhe a autonomia, a relação social e a identidade de seus alunos, bem como do aluno com autismo. "É fundamental, por conseguinte, que a concepção na educação seja centrada prioritariamente no ser humano e não na patologia" (Cunha, 2012, p. 53). Diante das atividades desempenhadas e articuladas Benitez et al. (2017), destaca que o professor necessita ter conhecimento sobre as características de cada aluno com TEA para a construção das aulas e sua a inclusão nas turmas.

\section{Considerações Finais}

No contexto educacional a criança com TEA necessita de várias orientações, auxílio e compreensão. É preciso que o ambiente esteja adaptado para atender suas demandas, fatos que facilitam e possibilitam o seu desenvolvimento.

Além da presença de um profissional capacitado, é preciso desenvolver ações para lidar com as especificidades exigidas por esse púbico. No entanto são necessárias mais intervenções de capacitação apropriadas para os docentes, a fim de obter ações e reações voltadas a uma inclusão em potencial, permitindo ao aluno autista além da socialização, um desenvolvimento pedagógico e comportamental efetivo.

Serra (2010) contribui apontando a presença de um mediador ou tutor para auxiliar o professor no processo de inclusão. O papel do tutor é de assessorar a criança autista nas atividades propostas, incentivando-a a participar nas atividades comuns à classe e quando isso não for possível, oferecer uma atividade paralela ao sujeito ou mesmo mudar de ambiente com ele. O tutor é também considerado uma ponte nas relações sociais entre o aluno autista e o grupo. Ainda para Amâncio e Assali (2005), o importante é desapegar-se de manuais pedagógicos e psicológicos, sem visar a uma educação homogênea, a fim de que o processo de inclusão não seja comprometido (Caneda; Chaves, 2015, p. 146).

Compreender os comportamentos das crianças com espectro autista vai de encontro com os contextos interativos, a mediação do adulto e, sobretudo, as particularidades de cada educando para o envolvimento de todos.

É válido destacar que o autismo é um transtorno complexo, portanto não existe uma terapia ou método isolado que atenderá as demandas da pessoa com TEA. É preciso fortalecer os vínculos entre família, escola, cuidadores e terapeutas para que deste modo, juntos possam criar uma melhor estratégia de intervenção para o ensino de habilidades que vise melhorar o desenvolvimento da criança.

Nesse viés, espera-se que o ensino estruturado como ferramenta de ensino baseado nas intervenções TEACCH, ABA e PECS se mostra promissor com a necessidade de mais estudos, no sentido de organizar o ambiente e as atividades para que a pessoa com TEA desenvolva potencialmente sua autonomia, a interação, comunicação, a cognição, bem como regular os comportamentos inadequados, e passe a ter uma compreensão a respeito do mundo que a cerca. 


\section{Referências}

Aires, A. C. S. (2014). Autismo: convívio escolar, um desafio para a educação. Anais VI FIPED... Campina Grande: Realize Editora, 2014. <https://www.editorarealize.com.br/index.php/artigo/visualizar/6283>

Almeida, A. J. F. \& Felizardo, S. (2015). Alunos com perturbações do espectro do autismo, interação com os pares e inclusão escolar: Percepções das crianças do $1^{\circ}$ ciclo do ensino básico trabalho de projeto de Educação Especial.

American Psychiatric Association (APA). (2014). Manual Diagnóstico e Estatístico de Transtornos Mentais - DSM V. Tradução de Maria Inês Correa Nascimento et al; revisão técnica Aristides Volpato Cordiolo. (5a ed.), Artes Médicas.

Assumpção, F. B., \& Pimentel, A. C. M. (2017). Autismo infantil. Revista Brasileira de Psiquiatria, $22(37), 9$.

Balestro, J. I. et al. (2012). Questionário sobre dificuldades comunicativas percebidas por pais de crianças do espectro do autismo. Revista da Sociedade Brasileira de Fonoaudiologia, 17(3), 279-286.

Brasil (2011). Decreto n 7.611, de 17 de novembro de 2011. Brasília, 2011. www.planalto.gov.br/ccivil_03/_ato2011-2014/2011/decreto/d7611.

Baptista, Cláudio Roberto. \& Bosa, Cleonice. (2012). Autismo e educação: reflexões e propostas de intervenção. Artmed.

Benitez, P.; Gomes, M., \& Bondioli, R. Domeniconi, C. (2017). Mapeamento das estratégias inclusivas para estudantes com deficiência intelectual e autismo. Psicologia em Estudo, 22(1), 81-93.

Bosa, C.; \& Callias, M. (2012). Autismo: breve revisão de diferentes abordagens. Psicologia: Reflexão e Crítica. 13(1).

Burtet, K. S. \& Godinho, L. B. R. (2017). Envolvimento familiar na clínica do autismo. Revista Cippus - UNILASALLE, 7(2).

Caneda, C. R. G. \& Chaves, T. M. L. (2015). A percepção do professor e do tutor frente à inclusão da criança com autismo no ensino regular. Aletheia 46(1): $142-158$.

Cunha, E. (2012). Autismo e inclusão: psicopedagogia e práticas educativas na escola e na família. (4a ed.).

Facion, José Raimundo. (2007). Transtorno do Desenvolvimento e do comportamento. IBPEX.

Glat, R. \& Nogueira, M. L. de L. (2016). A formação de professores para a Educação Inclusiva. Revista Comunicações, UNIMEP, $134-141$.

Gomes, A. N. \& Silva, C. B. da. 2007. Software Educativo para Crianças Autistas de Nível Severo. In: $4^{\circ}$ Congresso Internacional de Pesquisas em Design, Rio de Janeiro. www.anpedesign.org.br/artigos.

Kanner L. (2015). Autistic disturbances of affective contact. NervousChild. 1943. Papirus.

Kupfer, M. C. M. (2000). Notas sobre o diagnóstico diferencial da psicose e do autismo na infância. Psicol. USP, 11(1)

Lampreia, C. (2007). A perspectiva desenvolvimentista para a intervenção precoce no autismo. Estudos de Psicologia, 24(1), 105-114.

Leboyer, Marion. (2015). Autismo infantil: fatos e modelos. (5a ed.), Papirus.

Leon, Viviane Costa de. (2016). Práticas baseadas em experiências para aplicação do TEACCH nos Transtornos do Espectro do Autismo. Memnon.

Leon, Viviane Costa de. \& Moraes, Claudia. (2018). Portfólio de atividades para ensino estruturado do Autismo. Memnon.

Marinho, E. A. R. \& Merkle, V. L. B. (2009). Um Olhar Sobre O Autismo e sua especificação. XI Congresso Nacional de Educação - EDUCERE. III Encontro Sul Brasileiro de Psicopedagogia.

Mazzotta, Marcos José Silveira. (2009). Educação Especial no Brasil: história e políticas públicas. (5a ed.), Cortez.

Matsumoto, L. E. \& Campos, L. M. L. (2008). Favorecendo a cooperação entre crianças: relato de uma experiência. Revista Simbio-Logias, 1(1): 200-213.

Mello, Ana Maria Serrajordia de. (2011). Autismo: guia prático. (7a ed.), Corde.

Nilsson, I. (2014). Introdução a educação especial para pessoas com transtornos de espectro autístico e dificuldades semelhantes de aprendizagem. Congresso Nacional sobre a Síndrome de Autismo. http://www.ama.org.br/download/Autismo-IntrodEducEspecial.

Papim, A. A. P., \& Sanches, K. G. (2013). Autismo e Inclusão: levantamento das dificuldades encontradas pelo professor do atendimento educacional especializado em sua prática com crianças com autismo. 84 p. Monografia (Especialização) - Centro Universitário Católico Salesiano.

Pimentel, A. G. L. \& Fernandes, F. D. M. (2014). A perspectiva de professores quanto ao trabalho com crianças com autismo. Audiol Commun Res. 19(2): 171 $-178,2014$.

Pletsch, M. D. (2010). Repensando a inclusão escolar: diretrizes políticas, práticas curriculares e deficiência intelectual. Nau Edur.

Santos, A. J. \& Lemes, M. G. N. (2020). O espectro dos autismos e a psicose infantil: uma questão diagnóstica para a psicanálise. Revista Latinoamericana de Psicopatologia Fundamental, 23(2).

Uchôa, Y. F. (2015). A criança autista na educação infantil: desafios e possibilidades na educação inclusiva. UEP.

Ziraldo. (2013). Autismo: uma realidade. Editora Megatério estúdio. 